
\title{
R Research Square \\ PIWI-interacting RNAs are promising biomarkers for targeting glioma and other cancers
}

Omid Reza Tamtaji

Mohammad Behnam

Mohammad Ali Pourattar

Michael R Hamblin

Maryam Mahjoubin-Tehran

Hamed Mirzaei

Zatollah Asemi

\section{Video Byte}

Keywords: Cell Communication and Signaling, piRNAs, PIWI protein, glioma, apoptosis, migration, invasion, central nervous system, non-coding RNAs, P-element-induced wimpy testis, epigenetic regulation, transcription, translation, ping-pong cycle, metastasis, genome-wide association study, GWAS, tumorigenesis, glioblastoma, GBM

Posted Date: November 12th, 2020

DOI: https://doi.org/10.21203/rs.3.rs-106709/v1

License: (9) This work is licensed under a Creative Commons Attribution 4.0 International License.

Read Full License 


\section{Abstract}

Glioma is the most common primary brain tumor and represents a major health problem across the globe. Understanding how gliomas form has proven difficult, especially at the molecular level, but growing evidence points to the important roles played by non-coding RNAs, especially small non-coding RNAs that interact with PIWI proteins, or piRNAs. piRNAs execute functions associated with epigenetic reprogramming and can regulate transcription, translation, development, and mRNA stability. In fact, piRNAs have been detected in many types of cancer and are known to be involved in the development and spread of certain tumors. piRNAs are formed either through the "primary processing pathway" or the secondary "ping-pong cycle" pathway. In conjunction with PIWI proteins, piRNAs execute epigenetic regulation of genes by modifying histones. In this way, piRNAs can influence numerous molecular signaling pathways associated with the formation and spread of gliomas, including the PI3K/AKT and TNF signaling pathways. While more research is needed to understand how piRNAs can be detected and monitored in clinical settings, piRNAs are promising biomarkers for the diagnosis, prognosis, and treatment of glioma and other cancers. 